\title{
Graft lymphoma in a kidney transplant recipient: a case report
}

\author{
Ji-hyun Yeom ${ }^{1}$, Keun-Sang Kwon ${ }^{2}$, Hong Pil Hwang ${ }^{3}$, Hee Chul Yư ${ }^{3}$, Byeoung Hoon Chung ${ }^{3}$, Sung Kwang Park ${ }^{1}$, Sik Lee $^{1}$ \\ ${ }^{1}$ Department of Internal Medicine-Nephrology, Jeonbuk National University Hospital, Jeonju, Korea
${ }^{2}$ Department of Preventive Medicine, Jeonbuk National University Hospital, Jeonju, Korea
${ }^{3}$ Department of Surgery, Jeonbuk National University Hospital, Jeonju, Korea
}

Background: Post-transplant lymphoproliferative disorders (PTLDs) are significant complications in solid organ recipients, with various lymphoid and/or plasmacytic proliferations due to immunosuppression. Here, we present the case of pathologically confirmed lymphoma after nephrectomy in a KT recipient with allograft dysfunction showing symptoms like urinary tract infection. Case report: A 37-year-old female was admitted with an abrupt $39^{\circ} \mathrm{C}$ fever. She went on continuous ambulatory peritoneal dialysis 6 months after graft failure. Eight years ago, she underwent deceased donor kidney transplantation for end-stage renal disease due to immunoglobulin A (IgA) nephropathy. The immunosuppressants she was taking included tacrolimus, mycophenolate mofetil, and prednisolone. No episodes of acute rejection occurred. However, a graft biopsy was performed 4 years after the kidney transplant due to decreased kidney function and showed recurrent IgA nephropathy. The patient complained of intermittent gross hematuria, mild graft discomfort, and general weakness. On admission, laboratory investigations revealed hemoglobin $(5.5 \mathrm{~g} / \mathrm{dL})$, lactate dehydrogenase $(1,453 \mathrm{IU} / \mathrm{L})$, and high-sensitivity C-reactive protein $(187 \mathrm{mg} / \mathrm{L})$. Physical examination demonstrated tenderness in the graft area. Enhanced computed tomography showed graft swelling with soft tissue infiltration, and we immediately started treatment with antibiotics. Nevertheless, her fever did not come down, and she became weaker than before hospitalization. Finally, we performed a graft nephrectomy as it was considered a graft intolerance syndrome. The histologic findings showed atypical lymphoid cells infiltrating diffusely between the sclerotic glomerulus and atrophic renal tubules. In addition, the patient was also positive for CD20 and Epstein-Barr virus (EBV)-encoded small nuclear RNAs in situ hybridization, suggesting EBV-positive diffuse large B cell lymphoma.

Conclusions: In this case, graft lymphoma manifested as persistent severe anemia and an abruptly high fever. Therefore, even though transplant recipients return to dialysis after graft failure, PTLDs should be included as a differential diagnosis from infection with a high index of suspicion.

Corresponding author: Sik Lee

E-mail: kidney@jbnu.ac.kr

(c) The Korean Society for Transplantation

This is an Open Access article distributed under the terms of the Creative Commons Attribution Non-Commercial License (http://creativecommons.org/licenses/by-nc/4.0/) which permits unrestricted non-commercial use, distribution, and reproduction in any medium, provided the original work is properly cited. 